TWMS J. App. Eng. Math. V.4, No.1, 2014, pp. 86-91.

\title{
APPROXIMATE OPTIMALITY CONDITIONS
}

\author{
R. SAHRAOUI ${ }^{1}$, A. BEDDANI ${ }^{2} \S$
}

\begin{abstract}
We propose in this paper a systematic study which is a variational approach of approximate optimality conditions in terms of Ekeland's variational principle and some of its applications. Using a generalised differentiation(sub-differentiability) theory for non-smooth functions, new properties are then identified and approximate optimality conditions are established in the cases: convex, locally Lipschitz and finally lower semi-continuous.
\end{abstract}

Keywords: Sub-differential, Qualification condition, Convex analysis, Normal compacity, Variational principal.

AMS Subject Classification: 49J52, 90C56.

\section{INTRODUCTION}

The differential of a convex function generalizes the notion of derivative and provided its first examples in the theory of maximal monotone operators, so successful for partial differential equations and integral equations . Another motivation also is an adjunct of the work without convexity, called nonsmooth analysis. This paper is based on the limiting Fréchet sub-differential which is introduce by Mordukhovich for several reasons: in particular because this sub-differential is "smaller" than of Clarke see [2], it contains fewer errors and it is interesting for the riches their calculations. One objective of the optimization is to establish necessary and sufficient optimality conditions if possible. The existence of the optimum solution is ensured for example by the compactness of the domain and the semi-continuity of the objective. And if one of these conditions is not satisfied the problem may not have exact solutions, but if the objective is bounded from below, the infinimum exists without being hit. It is for this reason Ekeland thought to the notion of the approximate solution. And he was the first in 1974 which gave the necessary conditions of optimality for approximate solutions only for the case where the data of the problem are differentiable. This paper carries on the one hand on the Ekeland variational principle, has been very important in nonlinear analysis, in which he enjoyed a great variation of applications ranging from geometry Banach spaces see [1], in optimization theory see Ekeland [3],[4] and sub-differential generalized calculation to calculate the variation see [2]. And secondly on the nonsmooth analysis in order to generate optimality conditions for optimization problems with constraints by applying the variational principle of Ekeland to obtain the approximate optimality conditions in different situations.

\footnotetext{
${ }^{1}$ Laboratory of Pure and Applied Mathematics, Faculty of SEI,University of Mostaganem, Algeria, e-mail: Sahraouimosta@yahoo.fr

${ }^{2}$ University of Oran, Faculty of SEA, Department of Mathematics, Algeria, e-mail: beddanixabd@yahoo.fr

$\S$ Submitted for GFTA'13, held in Işık University on October 12, 2013. TWMS Journal of Applied and Engineering Mathematics, Vol.4, No.1; (c) Işılk University, Department of Mathematics 2014; all rights reserved.
} 


\section{The Classical optimality CONDitions}

In general, we are interested in optimization to the necessary and sufficient conditions in different situations. Let the minimisation problem:

$$
\begin{gathered}
(P)\left\{\begin{array}{lc}
\min f(x) \\
x \in D
\end{array},\right. \\
D \subset X \quad X \rightarrow \overline{\mathbb{R}} \\
D=\left\{x \in X / g_{i}(x) \leq 0, i=\overline{1, . . n}\right\}
\end{gathered}
$$

\subsection{Case: Data are differentiable.}

Theorem 2.1. If $f_{G}^{\prime}($.$) exist and a a local min of (P) \Longrightarrow\left\langle f_{G}^{\prime}(a), v\right\rangle \geq 0, \forall v \in T(D, a)$

2.1.1. Theorem of Kuhn-Tucker. [7]

Theorem 2.2. If $D$ is qualified and $a$ is a local min of $(P)$, then $\exists \lambda \in \mathbb{R}^{n}$ (positive):

$$
\left\{\begin{array}{l}
\text { i) } f^{\prime}(a)+\sum_{i=1}^{n} \lambda_{i} g_{i}^{\prime}(a)=0 \backslash \\
\text { ii) } \lambda_{i} g_{i}(a)=0, \forall i \in\{1, \ldots, n\} .
\end{array}\right.
$$

\subsection{Case: Data are convex.}

Definition 2.1. The sub-differential of $f$ at $\bar{x}$ is the following set, and denoted by:

$$
\partial f(\bar{x})=\left\{x^{*} \in X^{*} / f(x) \geq f(\bar{x})+\left\langle x^{*}, \bar{x}-x\right\rangle, \forall x \in X\right\} .
$$

\subsubsection{Theorem of Rockafellar. [7]}

Theorem 2.3. Let $f, D$ convex and $f$ continuous.

$a$ is local minimum of $(P) \Longrightarrow 0 \in \partial f(a)+N(D, a)$.

Theorem 2.4. Let $f, g_{i}$ convex continuous.

$a$ is local minimum of $(P) \Longrightarrow \exists \lambda_{i} \geq 0, i=0, \ldots . n$ :

$$
\begin{aligned}
& \text { i) } g_{i}(a) \leq 0 \text { and } \lambda_{i} g_{i}(a)=0, i=\overline{1, . ., n} . \\
& \text { 2i) } 0 \in \lambda_{0} \partial f(a)+\sum_{i=1}^{n} \lambda_{i} \partial g_{i}(a) .
\end{aligned}
$$

\subsection{Case: Data are locally Lipschitz.}

Definition 2.2. Let $f: X \rightarrow \overline{\mathbb{R}}$ such that the derivative of $f_{c}^{\prime}(a,$.$) exists. Clarke sub-$ differential of $f$ at $x$ is the set noted as follows:

$$
\partial_{C} f(x)=\left\{x^{*} \in X^{*} /\left\langle x^{*}, \nu\right\rangle \leq f_{c}^{\prime}(x, \nu) ; \forall \nu \in X\right\} .
$$

2.3.1. Theorem of Clarke. [2]

Theorem 2.5. Let $f$ locally Lipschitzian and a local minimum of $(P) \Longrightarrow$

$$
0 \in \partial_{c} f(a)+N_{c}(D, a)
$$

Theorem 2.6. Let $f, g_{i}$ locally Lipschitzian and a local minimum of $(P) \Longrightarrow \exists \lambda_{i} \geq 0$, $i=0, \ldots . n /$

$$
\left\{\begin{array}{l}
i) 0 \in \lambda_{0} \partial_{c} f(a)+\sum_{i=1}^{n} \lambda_{i} \partial_{c} g_{i}(a) \\
2 i) \lambda_{i}=0 \text { si } i \notin I(a)
\end{array}\right.
$$




\subsection{Case: Data are lower semi-continuous.}

Definition 2.3 (Fréchet normal cone). Let $C$ a sub set nonempty of a Banach space $X$ and $x \in \mathrm{clC}$, the following nonempty set:

$$
\hat{N}(C, x)=\left\{x^{*} \in X^{*} / \limsup _{y \rightarrow \infty} \frac{\left\langle x^{*}, y-x\right\rangle}{\|y-x\|} \leq 0\right\},
$$

is called the Fréchet normal cone of $C$ at $x$.

\subsection{Limiting Normal Cone.}

Definition 2.4. The nonempty set:

$$
N(C, \bar{x})=\limsup _{x \rightarrow \bar{x}} \hat{N}(\bar{x}, C),
$$

is called the limiting normal Cone at $\bar{x} \in \mathrm{clC}$.

\section{Remark 2.1.}

$$
\text { Wehave }: \hat{N}(C, \bar{x}) \subset N(C, \bar{x}), .
$$

for all set $C \subset X$ and all point $\bar{x} \in \mathrm{clC}$.

\subsection{Limiting Sub-Differential.}

Definition 2.5. Let $\bar{x} \in \operatorname{domf}$, we have:

$$
\begin{aligned}
& \text { i) } \partial f(\bar{x})=\left\{x^{*} \in X^{*} /\left(x^{*},-1\right) \in N((\bar{x}, f(\bar{x})) \text {,épi } f)\right\} \text {, } \\
& \text { 2i) } \partial^{\infty} f(\bar{x})=\left\{x^{*} \in X^{*} /\left(x^{*}, 0\right) \in N((\bar{x}, f(\bar{x})) \text {,épif })\right\} \text {. }
\end{aligned}
$$

are called respectively the limiting sub-differential and the singular sub-differential of $f$ at $\bar{x}$.

\subsection{Fréchet Sub-Differential.}

Definition 2.6. The Fréchet sub-differential of the function $f$ at $\bar{x}$ is defined by:

$$
\hat{\partial} f(\bar{x})=\left\{x^{*} \in X^{*} /\left(x^{*},-1\right) \in \hat{N}((\bar{x} ; f(\bar{x})), \text { épi } f)\right\},
$$

And we observe that $\hat{\partial} f(\bar{x}) \subset \partial f(\bar{x})$ for all $\bar{x} \in \operatorname{dom} f$. So the function $f$ says regular at $\bar{x}$ if $\hat{\partial} f(\bar{x})=\partial f(\bar{x})$.

Theorem 2.7. Let $X$ an Asplund space see[8] and a function $f: X \rightarrow \overline{\mathbb{R}}$ normally compact see[8] and lower semi continuous on $X$, then if $\bar{x}$ minimise $f$ on $X$ we have $0 \in \partial f(\bar{x})$.

\section{Ekeland's Variational Principle}

Theorem 3.1. [4] Let $(X,\|\|$.$) of Banach and f: X \rightarrow \overline{\mathbb{R}}$ a proper function, l.s.c. and bounded from below on $X$.

For each $\varepsilon>0$ and each $u \in X$ such that:

$$
f(u) \leq \inf _{X} f+\varepsilon .
$$

and for all $\lambda>0$, there exists $v \in X$, such that:

$$
\begin{gathered}
f(v) \leq f(u), \\
\|v-u\| \leq \lambda, \\
f(v)<f(x)+\frac{\varepsilon}{\lambda}\|x-v\|, \forall x \in X, \forall x \neq v .
\end{gathered}
$$




\section{Approximate Optimality Conditions}

\subsection{The Differentiable Case.}

Theorem 4.1. Let $X$ a Banach space and $f: X \rightarrow \overline{\mathbb{R}}$ a function l.s.c, Gteaux differentiable and bounded from below on $X$. Then: $\forall \epsilon>0, \forall u \in X$ verified $f(u) \leq \inf _{x \in X} f(x)+\varepsilon$ et $\forall \lambda>0$, there exists $v \in X$ such that

$$
\begin{gathered}
f(v) \leq f(u), \\
\|v-u\| \leq \lambda, \\
\left\|f_{G}^{\prime}(v)\right\|^{*} \leq \frac{\epsilon}{\lambda} .
\end{gathered}
$$

Let $X$ a Banach space, $D$ be a closed sub set of $X$ and a function $f: X \rightarrow \overline{\mathbb{R}}$.

consider the problem

$$
\left(P_{1}\right)\left\{\begin{array}{c}
\inf f(x) \\
x \in D
\end{array}\right.
$$

Theorem 4.2. Assumed $f$ l.s.c., Fréchet differentiable on $X$ and bounded from below on $D$. Then: $\forall \varepsilon>0, \forall u \in X$ verified $f(u) \leq \inf _{x \in D} f(x)+\varepsilon$, and $\forall \lambda>0$, there exists $x_{\epsilon} \in D$ such that

$$
\begin{gathered}
f\left(x_{\varepsilon}\right) \leq f(u), \\
\left\|u-x_{\varepsilon}\right\|<\lambda, \\
\left\langle f_{F}^{\prime}\left(x_{\varepsilon}\right), v\right\rangle+\frac{\varepsilon}{\lambda}\|v\| \geq 0, \forall v \in T\left(D, x_{\varepsilon}\right) .
\end{gathered}
$$

Let $X$ a Banach space, $D \subset X$ and $f: X \rightarrow \overline{\mathbb{R}}$ Fréchet differentiable. consider the problem $\left(P_{2}\right)$ type:

$$
\left(P_{2}\right)\left\{\begin{array}{c}
\inf f(x) \\
x \in D
\end{array} \quad \text { with } D=\left\{\begin{array}{c}
x \in X / g_{i}(x)=0, \forall i \in \overline{1, \ldots, p}, \text { and } \\
g_{i}(x) \leq 0, i=\overline{p+1, . ., n}
\end{array}\right\}\right.
$$

$\mathrm{g}_{i}(i=\overline{1, . ., n})$ are $C^{1}$ class in sense of Fréchet and the domain $D$ is qualified.

Theorem 4.3. If the function $f$ is bounded from below on the domain $D$, then: $\forall \epsilon>0, \exists x_{\epsilon} \in D$ such that $f\left(x_{\epsilon}\right) \leq \inf _{x \in D} f(x)+\varepsilon^{2}, \exists \lambda_{i} \in \mathbb{R}^{+}, i \in \overline{1, \ldots, n}$ verified:

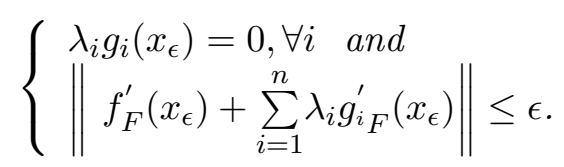

4.2. The Convex Case. Let $X$ a Banach space, $D$ a closed sub set of $X$ and $f: X \rightarrow \overline{\mathbb{R}}$ is convex in the problem $\left(P_{1}\right)$.

Theorem 4.4. Let $X$ a Banach space and $f: X \rightarrow \overline{\mathbb{R}}$ l.c.i, proper, convex and bounded from below on $D$. Then:

$\forall \epsilon>0, \forall u \in X$ such that:

$\forall \lambda>0$ there exists $x_{\epsilon} \in D$, such that

$$
f(u) \leq \inf _{x \in D} f(x)+\epsilon, \text { and }
$$

$$
0 \in \partial f\left(x_{\epsilon}\right)+\frac{\epsilon}{\lambda} B^{*}+N\left(D, x_{\epsilon}\right) .
$$

Let $X$ a Banach space, $D \subset X$ and $f: X \rightarrow \overline{\mathbb{R}}$ convex in the problem $\left(P_{2}\right)$ with $g_{i}(i=\overline{1, . ., n})$ are convex. 
Theorem 4.5. If $f: X \rightarrow \overline{\mathbb{R}}$, l.s.c., proper and bounded from below and let $\epsilon>0, u \in D$, such that

$$
f(u) \leq \inf _{D} f(x)+\varepsilon, \text { and }
$$

$\forall \lambda>0$, there exists $x_{\epsilon} \in D$ and $\exists\left(\lambda_{0}, . ., \lambda_{n}\right) \in \mathbb{R}_{+}^{n}$ verified

$$
0 \in \lambda_{0} \partial f\left(x_{\epsilon}\right)+\sum_{i=1}^{n} \lambda_{i} \partial g_{i}\left(x_{\varepsilon}\right)+\lambda_{0} \frac{\varepsilon}{\lambda} B^{*} .
$$

\subsection{The Locally Lipschitz Case.}

Theorem 4.6. Let $X$ a Banach space, $D$ a closed sub set of $X$ and $f: X \rightarrow \overline{\mathbb{R}}$ l.s.c., locally Lipschitzian and bounded from bellow. Then:

$\forall \epsilon>0, \forall u \in X$ such that:

$$
f(u) \leq \inf _{x \in D} f(x)+\varepsilon, \text { and }
$$

$\forall \lambda>0$, there exists $x_{\epsilon} \in D$, verified

$$
0 \in \partial_{c} f\left(x_{\epsilon}\right)+\frac{\varepsilon}{\lambda} B^{*}+N_{c}\left(D, x_{\epsilon}\right) .
$$

Let $f, g_{i}(i=\overline{1, . ., q})$ and $h_{j}(j=\overline{1, . ., p})$ locally Lipchitzian in $\left(P_{2}\right)$

Theorem 4.7. Let $f: X \rightarrow \overline{\mathbb{R}}$, l.s.c., proper and bounded from bellow. Then $\forall \epsilon>0$, and $\forall u \in D$, such that:

$$
f(u) \leq \inf _{D} f(x)+\epsilon, \text { and }
$$

$\forall \lambda>0$, there exists $x_{\epsilon} \in D \exists\left(\lambda_{0}, . ., \lambda_{n}\right) \in \mathbb{R}_{+}^{n}$ verifiant:

$$
0 \in \lambda_{0} \partial f\left(x_{\varepsilon}\right)+\sum_{i=1}^{q} \lambda_{i} \partial_{c}\left|g_{i}\left(x_{\varepsilon}\right)\right|+\sum_{i=1}^{p} \lambda_{i} \partial_{c} h_{i}\left(x_{\varepsilon}\right)+\frac{\varepsilon}{\lambda} B^{*} .
$$

\subsection{The Lower Semi Continuous Case.}

Theorem 4.8. Let $X$ an Asplund space see [8] and $f: X \rightarrow \overline{\mathbb{R}}$ normally compact see [8], l.s.c. proper and bounded from below. Then:

$\forall \epsilon>0$ and $\forall u \in X$ such that

$$
f(u) \leq \inf _{x \in X} f(x)+\epsilon, \text { and }
$$

for all $\lambda>0$, there exists $x_{\varepsilon} \in X$, verified:

$$
0 \in \partial f\left(x_{\varepsilon}\right)+\frac{\epsilon}{\lambda} B^{*}
$$

Theorem 4.9. Let $X$ an Asplund space, $D$ a closed sub set of $X$ and $f: X \rightarrow \overline{\mathbb{R}}$ normally compact, l.s.c. and bounded from bellow. Then:

$\forall \epsilon>0$ and $\forall u \in X$ such that:

$$
f(u) \leq \inf _{x \in D} f(x)+\varepsilon, \text { and }
$$

$\forall \lambda>0$, there exists $x_{\epsilon} \in D$, verified:

$$
0 \in \partial f\left(x_{\epsilon}\right)+\frac{\varepsilon}{\lambda} B^{*}+N\left(D, x_{\epsilon}\right)
$$

Remark 4.1. This theorem is a general result, it gives the theorem (4.2) in the differentiable case, Theorem (4.4) in the convex case and Theorem (4.6) in the case of locally Lipschitz. 


\section{REFERENCES}

[1] Borwein, J.M., Preiss, D.,(1987), A smooth variational principle with applications to subdifferentiability and to differentiability of convex functions, Trans. Amer. Math. Soc. 303, p. 517-527.

[2] Clarke, F.H., (1975), Generalized Gradients and Applications, Volume 205, p. 247-262.

[3] Ekeland, I., (1979), Nonconvex minimization problems, Volume 1, Number 3, p. 443-473.

[4] Ekeland, I., (1974), On the variational principle, J. Math. Anal. Appl. 47, p. 324-353.

[5] Mordukhovich, B. S., (2006), Variational Analysis and Generalized Differentiation I and II, Springer, New-York.

[6] Mordukhovich, B.S., (2004), Variational and Nonsmooth analysis, Departement of Mathematics Wayne State University, Presented at the Summer School of the First ICCOPT.

[7] Rockafellar, R.T., (1972), Convex Analysis, Princeton, New Jersey. Princeton University.

[8] Sahraoui, R., Thibault, L., (2008), Bolza type problem in discrete time, Taiwanese J. Math., vol.12, No.6, p. 1385-1400. 